\title{
The Astronaut Game - A Vocabulary Game Employing Natural Language Processing Techniques
}

\author{
Irina Toma, Bianca-Eugenia Stefan, Mihai Dascalu, Stefan Trausan-Matu \\ University Politehnica of Bucharest \\ 313 Splaiul Independenței, Bucharest, Romania \\ \{irina.toma, mihai.dascalu, stefan.trausan\}@upb.ro, bianca.stefan1996@gmail.com
}

\begin{abstract}
Serious games are viable alternatives for learning and for acquiring new skills. Serious games introduce users with a learning environment that mimics real life situations, where they can practice and improve their skills. While considering education, we focus on games used for language learning, which cover multiple categories that range from vocabulary acquisition, comprehension, collaboration, to free text writing. The serious game described in this paper - the Astronaut Game - is design for English as Second Level users and consists of two parts. The first part consists of a Platform Jumper for vocabulary acquisition, in which an astronaut runs and jumps to collect rocket pieces that symbolize words; the aim of this sub-game is to teach words from different lexical fields to the players, depending on the level they are playing. The second part, the Rocket Hangman, is centered on comprehension and users must identify the central keywords from the reference text. Our game was tested by a group of 16 individuals and received positive feedback.
\end{abstract}

\section{Author Keywords}

Serious Games; Language Learning; Vocabulary

Acquisition; Comprehension; ReaderBench framework

\section{ACM Classification Keywords}

I.2.1 [Applications and Expert Systems]: Games.

I.2.7 [Natural Language Processing]: Discourse, Language parsing and understanding, Text analysis.

\section{General Terms}

Natural Language; Web-based Interaction; Games; Language acquisition; Text analysis.

DOI: $10.37789 /$ rochi.2021.1.1.11

\section{INTRODUCTION}

Serious games are gaining popularity as they are attractive, while integrating educative content with entertaining aspects of games. Even though the words "serious" and "games" have opposing meanings at surface level, many scholars acknowledge them as engaging and purposeful [8]. These games stimulate the motivation to learn through quests and rewards that divert the attention from the possible stress or pressure encountered when studying. Schwabe et al. [9] state that learning in stressful environments reduce students' performance by $30 \%$ because stress affects memory encoding.
The applications of serious games are vast and target different markets, including education, military, healthcare, politics, art, religion, etc. Serious games include more than computer games - for example, military environment games have evolved from rule-based games to complex battle and flight simulators, which help soldiers improve their tactical skills and provide a safe environment to practice different routines. In education, serious games teach students various concepts, such as historical events, cultural differences, physics, or new languages. Since serious games in education represent a broad concept, our research group is focused on games used to learn English as a second language.

\section{CREATING GOOD SERIOUS GAMES}

Serious games have both educational and entertaining characteristics. The Summit of Educational Games [2] presented a list of features that serious games should address in order to obtain the desired educational outcome(s). From this list, several features can be applied to the current research domain - i.e., learning a foreign language. The first feature states that serious games should have clear learning goals, as users should know why they are learning about a concept and how they can use it in real life. The games should present information personalized for the users' knowledge level and adapted to the situation in which the acquired knowledge is used. For example, stewards should know basic conversational terms, the meaning of safety instructions, etc.

Second, the applications should encourage users to learn more, either by seeking the information themselves, or by providing a controlled environment to accommodate discussions, ask questions, or provide insights. This can be achieved through forums, guided discussion groups, Q\&A or frequently asked questions sections, etc.

Third, another feature refers to monitoring the progress of users and providing feedback for their actions. Feedback can be provided through levels, badges, trophies, achievements, or scores. At an overarching level, the monitoring process can be extended to give overall statistical information to high-level users (e.g., teachers, parent, or administrators) to better monitor the progress of each student.

Lastly, all serious games should capture users' attention and keep them motivated to play the game, even if they failed at one step. Users should be allowed to finish the tasks in a reasonable amount of time, and they should be provided with 
a tutorial for the game, as well as hints through the game to help beginners overcome their personal challenges.

\section{LEARNING ENGLISH THROUGH SERIOUS GAMES}

Learning a new language is a long-term process that requires dedication and commitment from students, as well as patience and resourcefulness from teachers. At the beginning of the learning process, students are overwhelmed by the amount of information they must assimilate and tend to lose confidence in their abilities. To overcome this, the learning process should be closely monitored by a teacher, providing guidance, and learning materials. Since all students are different and they have different learning curves, the process of manually personalizing the materials and providing individual guidance is time and resource consuming. This is backed by a statistical study provided by Eurostat [1] which states that on average 12 pupils are assigned per teacher in upper secondary education in the European Union; the previous value is increased to 14 pupils per teacher in Romania.

The serious games developed in our research group come to support both students and teachers, as they provide educational content fitted for the individual needs of the students, progress tracking, challenges, and hints through the games, altogether in user-friendly interfaces. Up to this point, the developed serious games are organized in the following categories:

- Vocabulary acquisition games - this is the first phase of the learning process focused on assimilating individual words or simple phrases. The serious games included in this category are Semantic Boggle [11], Semantic Taboo [10] and LearnerSkill [3];

- Comprehension - in this phase, students enhance their ability to learn from different text materials. This category includes Edutainment Platform [13] and Reading Space Secrets [12];

- Collaboration - in this phase students improve their communication and collaboration skills by playing problem-solving games in a chat-like interface. The Robbers and The Others [14] is the representative game from this category;

- Free text writing - this is the last phase of the language learning process, where students feel confident enough to write essays on a topic given by their teacher. Their essays are evaluated, and suggestions are provided in terms of diacritics (for Romanian language), morphology, syntax, and semantics. Representative for this category is ReadME [15] as an Automated Writing Evaluation system.

The vocabulary acquisition phase is probably the most important step in learning a new language, as all other steps are based on the lexicon known by the user. Therefore, our research group implemented considerably more games in this area to provide diversity for a monotonous task and to give users the option to choose their preferred game. The game described in the next chapter contains two parts: the first one fits the vocabulary acquisition phase, while the second one is centered on comprehension.

\section{THE ASTRONAUT GAME}

The game developed in GameMaker Studio 2 (https://www.yoyogames.com/en/gamemaker) presents an astronaut from Earth whose ship crashed during one of his missions, resulting in his isolation on an unknown planet. In the crash, various parts of the rocket were scattered around the foreign land. Now, he needs the player's help to gather the pieces and assemble them to get back to Earth.

The first scene of the game begins with a short sequence telling the backstory of the main character. Afterwards, the player can choose which game to play, namely: a) Platform Jumper, which introduces words from different lexical fields, or b) Rocket Hangman, where users must guess the topic of a text. The game ends when all levels of the Platform Jumper were completed. The final scene displays the gathered rocket components and enables the rocket repair sequence.

The game is designed to be played completely in one session. The number of games and levels is small to keep the attention rate high. Because there is a relatively large number of lexical fields per level of complexity, the game levels slightly change every time; thus, the player benefits from a new experience with each gameplay.

\section{Platform Jumper}

Platform Jumper teaches words from different lexical fields to the players, depending on the level they are playing. In the current version, there are five levels that can be played, each in two stages. In the first phase, an astronaut runs and jumps to collect rocket pieces that symbolize the words prompted in the next stage (see Figure 9). Each level has a different layout and design of the platforms, but the purpose is the same: collect all pieces and advance to the next stage of the level. 


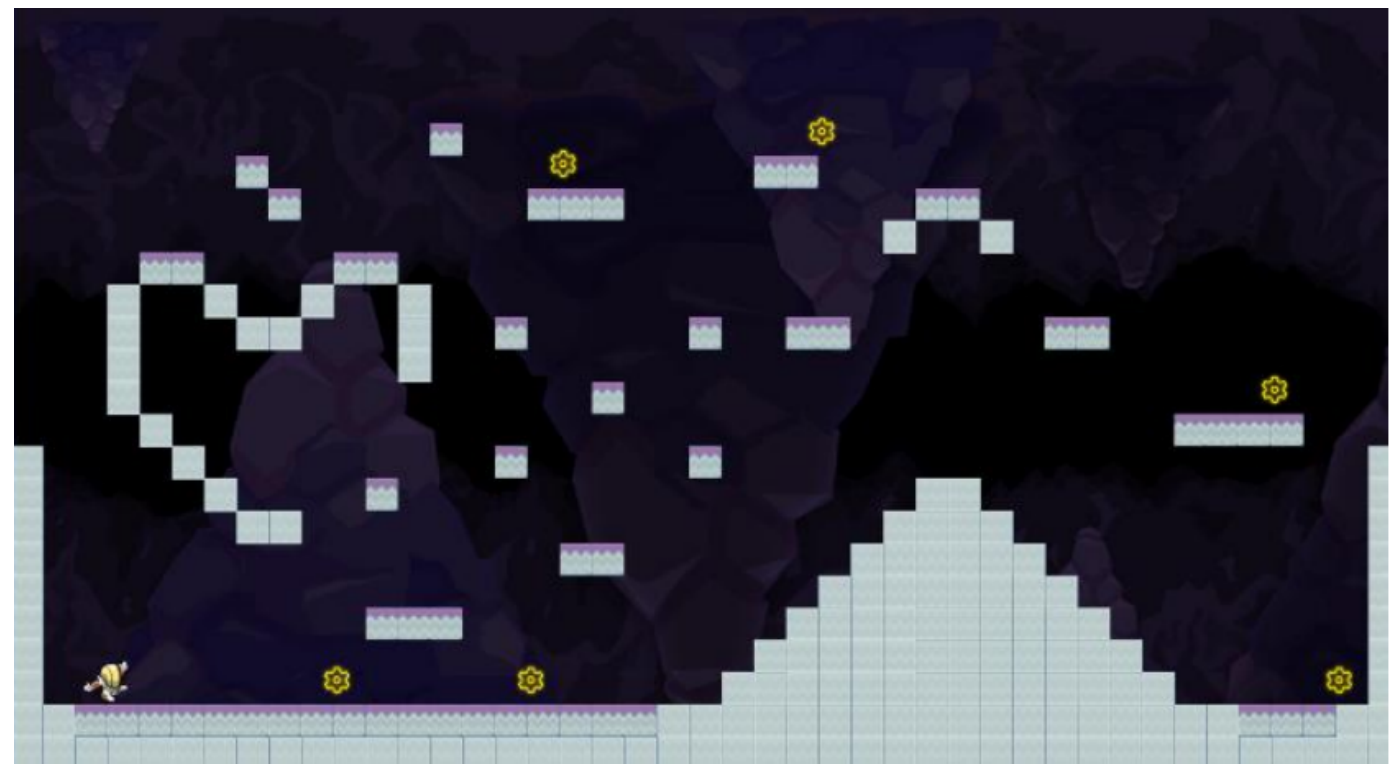

Figure 9. Platform Jumper interface - phase 1.

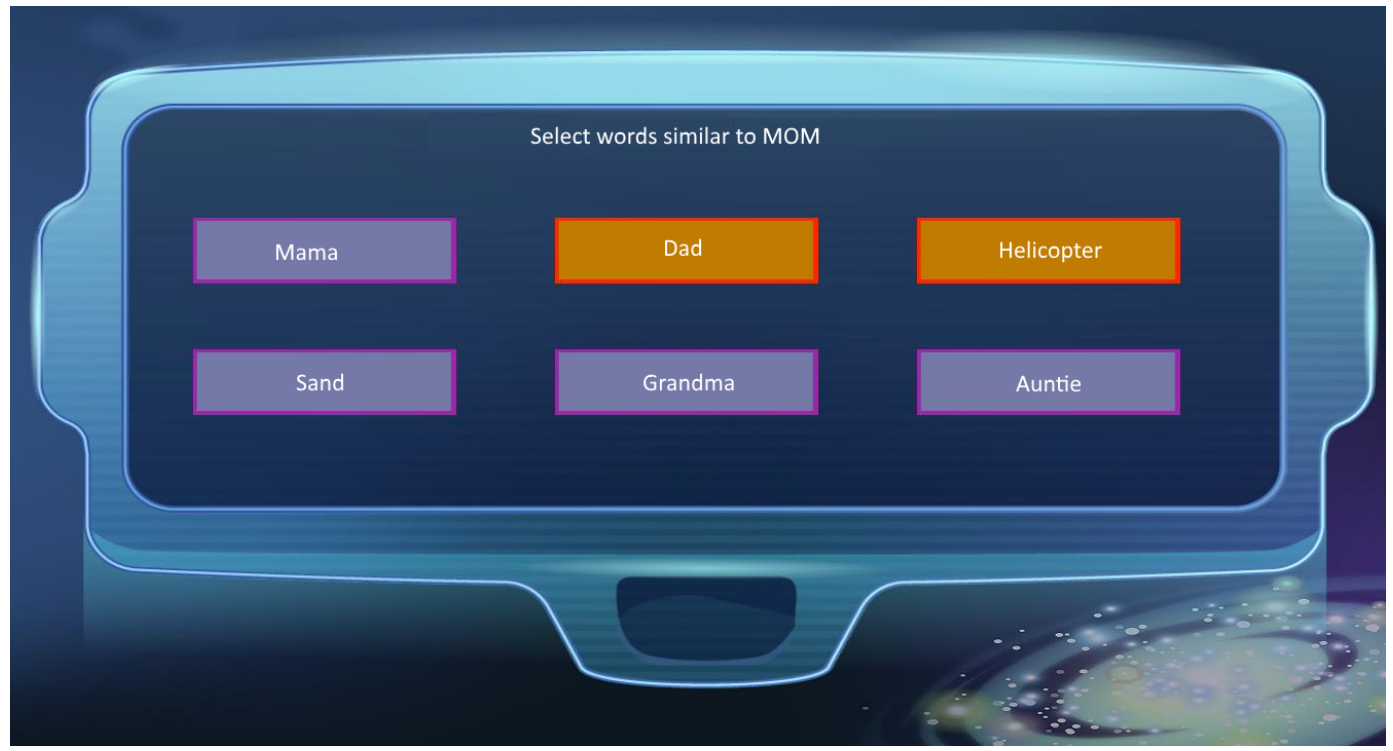

Figure 10. Platform Jumper interface - phase 2.

In the second phase, the lexical field selection, players are prompted 6 words from which they must choose the ones they believe are semantically related to another given word - they are either synonyms or refer to the same concept (see Figure 10).

The words displayed in the Platform Jumper interface are generated based on the Age of Acquisition (AoA) [6] list which contains over 31.000 words, together with their corresponding values that reflect the average age at which a word's meaning is properly acquired. Based on the Kuperman values, the words are divided into twenty groups.
For each group, semantic similarity, calculated as as $\mathrm{Wu}-$ Palmer distance [16] applied on WordNet [7], is computed between every two words, and only pairs with a semantic similarity higher that an imposed threshold (empirically set in our experiments at .37) are kept. The pairs are afterwards grouped around a common word and sorted ascendingly by their Kuperman value. For each group, a similarity matrix is built that is further used to apply affinity propagation clustering [5]. Therefore, we obtain a variable number of clusters that contain words of similar difficulty (i.e., comparable AoA scores) from the same lexical field. 


\section{Rocket Hangman}

The second serious game is a comprehension game, where users must guess the topic of a given text. As seen in Figure 11 , the left side of the interface displays a functional cartoon rocket, and the right side displays the text to be analyzed by the user. Below the text, the user inputs the guessed keyword that best describes the reference text. For every wrong guess, the user loses, one by one, parts of the rocket: the ignition burst of fire, the fins, the engine, followed by the main body. If users do not find the keyword before losing all the rocker pieces, they lose the game; nevertheless, the correct keyword is displayed on the screen for stimulating the learning process. The topic of the text is calculated using the ReaderBench API [4], a framework for advanced Natural Language Processing techniques, that extracts the most relevant concepts from a given text.

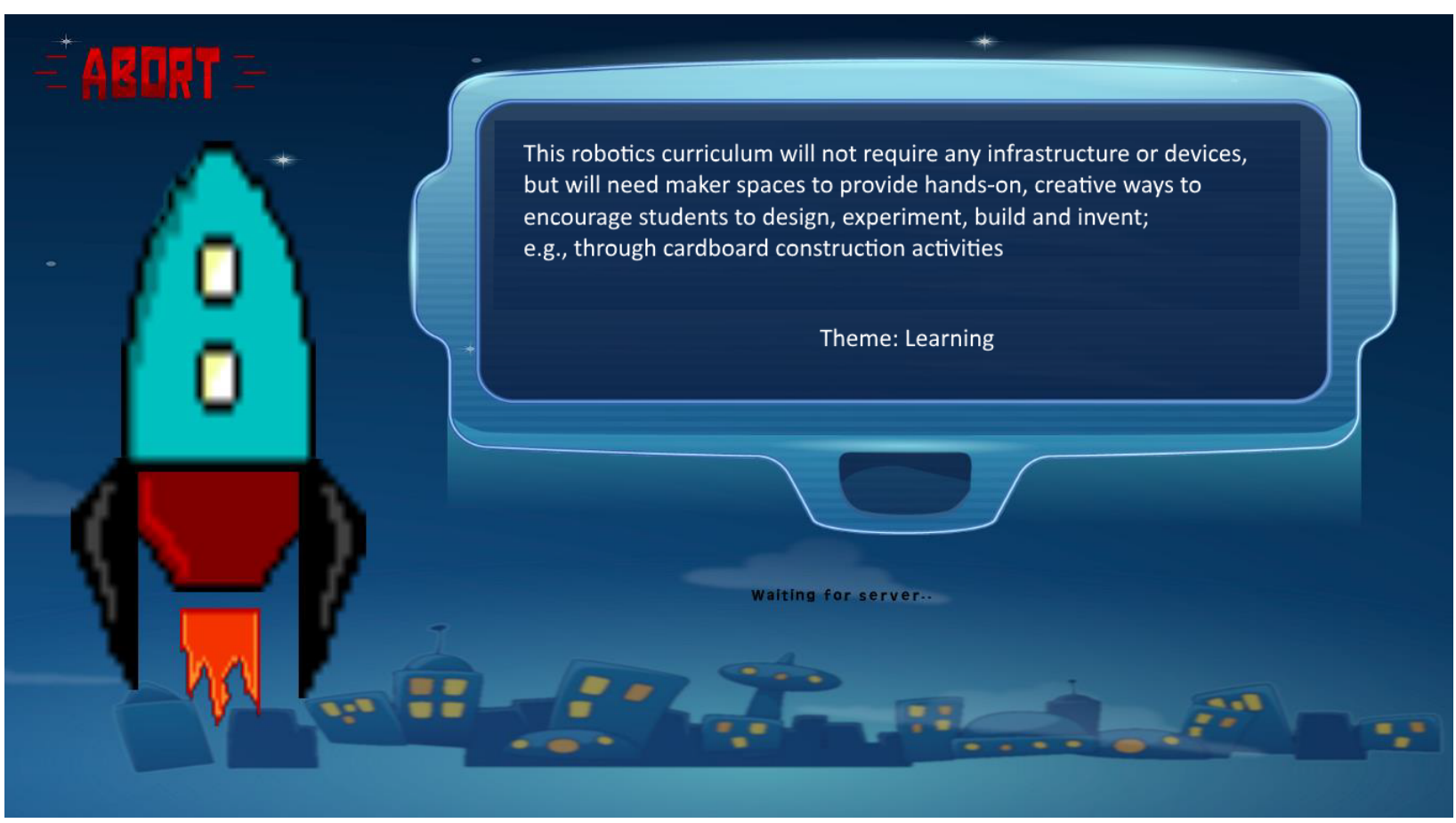

Figure 11. Rocket Hangman interface.

\section{RESULTS}

The game was tested by a group of 16 people, divided by their age and education level into four groups: $7-14$ years old (middle and elementary school - referred to as children), $15-18$ years old (high school - referred to as teenagers), 19 -25 years old (university - referred to as students), and over 25 years old (working adults - referred to as adults). Each age group consisted of four people, for an equal distribution of scores. Each user was asked to complete a survey on the gameplay with 4 questions having ratings on a 5-point Likert scale ( 1 - completely disagree; 5 - completely agree), as well as to provide feedback on the improvements they would like to see in the game.

The best score $(\mathrm{M}=4.75)$ was obtained for game navigation. Most users found it easy to switch between games and levels and were not in a situation where they did not know what to do next. All adults ranked this as 5 out of 5 . The theme of the game scored 3.62 out of 5 , with children and teenagers being more interested in the theme than the students and the adults. Children answered with an average of 4.25 , while the adults scored lower on average $(M=3.25)$. The difficulty of the game was evaluated with high discrepancies among the age groups. Children found the last levels of the Platform Jumper to be a little too difficult to solve, ranking it on average as 2.75. In contrast, adults gave a score of average 4.25. This outcome was expected as the complexity of the words in the final levels of the Platform Jumper correspond to age 12 (in accordance with the Age of Acquisition list), and two of the 4 children were younger than 12-years old. The last question reflects the probability of a user to play the game again. The participants gave a score of 3.87 out of 5 , which means most were convinced to replay it.

In terms of feedback, the subjects explained that they enjoy the layout of the platforms and the reverse hangman idea, but they would enjoy improvements in terms of graphics, as well as introducing music and sounds to the game. Moreover, people had different opinions regarding the difficulty level of the game. Children pointed out that the last levels were too difficult for them, teenagers considered the difficulty moderate, while the other two groups said the levels were too easy to solve. Altogether, they asked for more levels, a 
personalized difficulty level, as well as a high score to compete with their friends.

We also introduced a workaround to circumvent the rather long response time (on average 7 seconds) for the ReaderBench API to respond to a request having a long text as input. This downside was balanced by the fact that this game requires the player to first read a text, and then guess the keyword. In this situation, most players were too busy reading the text to notice the informing message about the game waiting on a response from the server. By the time the player tried to input his answer, the game would already have the keyword ready. This detail proved to help the dynamics of the game because it prevents players from randomly guessing, without first reading the text.

\section{CONCLUSIONS}

Serious games are an alternative to the standard learning process as they stimulate learning through quests and rewards that divert the attention from the possible stress or pressure students might face while studying. Several serious games have been developed in the current research group focusing on learning English as a second language. These games are divided in the following categories: vocabulary acquisition, comprehension, collaboration, and free text writing. The game presented in this paper, Astronaut game, is split into two parts, Platform Jumper that falls in the vocabulary acquisition category, and Rocket Hangman, which is a comprehension game.

Our Astronaut game was tested by a group of 16 individuals and received positive reviews. As a future implementation step, the feedback suggested by the users, such as improving the graphics and adding music and sounds, will be considered. We also consider adding more levels to the game, as well as adaptability of game level difficulty to better match the user knowledge based on an a priori evaluation test.

\section{ACKNOWLEDGMENTS}

The work presented in this paper was supported by an internal UPB Program - Proof of Concept.

\section{REFERENCES}

1. Eurostat, 2019. Ratio of pupils and students to teachers and academic staff by education level and programme orientation. Retrieved $15.06 \quad 2021$ from https://ec.europa.eu/eurostat/web/products-eurostatnews/-/DDN-20190911-1.

2. Federation of American Scientists, 2006. Harnessing the power of video games for learning: Report of the October 2005 Summit on Educational Games. Federation of American Scientists, Washington, DC, USA.

3. Grigoroiu, A.M., Toma, I., Dascalu, M., and TrausanMatu, S., 2017. LearnerSkill - A Serious Hex Wars Game Centered on Learner Comprehension. In Proceedings of the International Conference on Human-Computer Interaction (RoCHI 2017) (Craiova, Romania), MATRIX ROM.

4. Gutu, G., Dascalu, M., Trausan-Matu, S., and Dessus, P., 2016. ReaderBench goes Online: A ComprehensionCentered Framework for Educational Purposes. In Proceedings of the International Conference on HumanComputer Interaction (RoCHI 2016) (Iasi, Romania), MATRIX ROM, 95-102.

5. Hdbscan, 2016. Comparing Python clustering algorithms Retrieved $\quad 16.06 \quad 2021$ from https://hdbscan.readthedocs.io/en/latest/comparing clust ering algorithms.html.

6. Kuperman, V., Stadthagen-Gonzalez, H., and Brysbaert, M., 2012. Age-of-acquisition ratings for 30,000 English words. Behavior research methods 44, 4, 978-990.

7. Miller, G.A., 2010. WordNet Princeton University, Princeton, New Jersey, USA.

8. Ritterfeld, U., Cody, M., and Vorderer, P., 2009. Serious games: Mechanisms and effects Routledge, New York, NY \& London, UK.

9. Schwabe, L., Joëls, M., Roozendaal, B., Wolf, O.T., and Oitzl, M.S., 2012. Stress effects on memory: an update and integration. Neuroscience \& Biobehavioral Reviews $36,7,1740-1749$.

10.Toma, I., Alexandru, C., Dascalu, M., Dessus, P., and Trausan-Matu, S., 2017. Semantic Taboo - A Serious Game for Vocabulary Acquisition. Revista Romana de Interactiune Om-Calculator 10, 2, 241-256.

11.Toma, I., Alexandru, C.-E., Dascalu, M., Dessus, P., and Trausan-Matu, S., 2017. Semantic Boggle: A Game for Vocabulary Acquisition. In Proceedings of the 12th European Conference on Technology Enhanced Learning (EC-TEL 2017) (Tallinn, Estonia), Springer, 606-609.

12.Toma, I., Andrioaie, I., Dascalu, M., and Trausan-Matu, S., 2016. Reading Space Secrets - A Serious Game Centered on Reading Strategies. Revista Romana de Interactiune Om-Calculator 9, 4, 269-284. 
13.Toma, I., Bacioiu, F., Dascalu, M., and Trausan-Matu, S., 2018. The Edutainment Platform: Interactive Storytelling Relying on Semantic Similarity. In Proceedings of the Int. Conf. on Smart Learning Environments (ICSLE 2018) (Beijing, China), Springer, 87-96.

14.Toma, I., Brighiu, S.M., Dascalu, M., and Trausan-Matu, S., 2018. The Robbers and the Others - a Serious Game using Natural Language Processing. In Proceedings of the 3rd Int. Conf. on Smart Learning Ecosystems and Regional Development (SLERD 2018) (Aalborg, Denmark), Springer, 159-164.
15.Toma, I., Cotet, T.-M., Dascalu, M., and Trausan-Matu, S., 2019. ReadME - Your Personal Writing Assistant. In Proceedings of the 14th European Conference on Technology Enhanced Learning (EC-TEL 2019) (Delft, Netherlands), Springer, 751-755.

16.Wu, Z. and Palmer, M., 1994. Verb semantics and lexical selection. In Proceedings of the 32nd Annual Meeting of the Association for Computational Linguistics, ACL '94 (New Mexico, USA), ACL, 133-138. 